\title{
Statistical structures on the tangent bundle of a statistical manifold with Sasaki metric
}

\author{
Vladimir Balan*1 (D), Esmaeil Peyghan² (D), Esa Sharahi² ${ }^{2}$ \\ ${ }^{1}$ Department Mathematics-Informatics, Faculty of Applied Sciences, University Politehnica of Bucharest, \\ Bucharest RO-060042, Romania \\ ${ }^{2}$ Department of Mathematics, Faculty of Science, Arak University, Arak 38156-8-8349, Iran
}

\begin{abstract}
The first part of the paper is devoted to the classification of the statistical structures which live on the tangent bundle of a statistical manifold endowed with a Sasaki metric. Further, considering a Kähler structure on the base statistical manifold, we introduce a family of almost complex structures on the tangent bundle equipped with the Sasaki metric, and find equivalent conditions for which this family induces a Kähler structure. Finally, we derive equivalent conditions for existence of holomorphic structures on the tangent bundle equipped with the Sasaki metric in the presence of a statistical structure. Several illustrative examples are provided, as well.
\end{abstract}

Mathematics Subject Classification (2010). 53B05, 53B30, 53A15

Keywords. Holomorphic statistical manifold, statistical structure, Kähler structure, Sasaki metric

\section{Introduction}

Information geometry is a very effective branch of science, being regarded as a combination of differential geometry and statistics. Its applications can be found in various fields of research, like (see $[1,3,4,6,7,17]$ ): image processing, physics, computer science and machine learning. It merges fundamental differential geometry tools like metric, curvature and geodesics, to statistical models, and makes it possible to illustrate statistical objects as geometric ones. Moreover, the geodesics, and the totally geodesic submanifolds, in the presence of an affine connection, are natural generalizations of straight lines and affine subspaces, respectively, in the Euclidean space. Hence, conceptualizing statistical spaces in such a geometrical context, makes it convenient to study the statistical behaviors in depth. This kind of approach is based on the fact that geometrical objects are invariant under coordinate transformations. A detailed survey on information geometry can be found in [2].

\footnotetext{
*Corresponding Author.

Email addresses: vladimir.balan@upb.ro (V. Balan), e-peyghan@araku.ac.ir (E. Peyghan), esasharahi@gmail.com (E. Sharahi)

Received: 06.08.2018; Accepted: 28.09.2018
} 
Let $\Theta$ be an open subset of $\mathbb{R}^{n}$. We call statistical model, a set $S$ of probability density functions on a sample space $\Omega$ with parameter $\theta=\left(\theta^{1}, \cdots, \theta^{n}\right)$, such that

$$
S=\left\{p(x ; \theta): \int_{\Omega} p(x ; \theta)=1, \quad p(x ; \theta)>0, \quad \theta \in \Theta \subseteq \mathbb{R}^{n}\right\} .
$$

For a statistical model $S$, the semi-definite Fisher information matrix $g(\theta)=\left[g_{i j}(\theta)\right]$ is defined as

$$
\begin{aligned}
g_{i j}(\theta):= & \int_{\Omega} \partial_{i} \ell_{\theta} \partial_{j} \ell_{\theta} p(x ; \theta) \mathrm{d} x \\
& =E_{p}\left[\partial_{i} \ell_{\theta} \partial_{j} \ell_{\theta}\right],
\end{aligned}
$$

where $\ell_{\theta}=\ell(x ; \theta):=\log p(x ; \theta), \partial_{i}:=\frac{\partial}{\partial \theta^{i}}$, and $E_{p}[f]$ is the expectation of $f(x)$ with respect to $p(x ; \theta)$. Considering such kinds of information matrices, $S$ is also called an infomanifold (or statistical manifold). Relation (1.1) was the first occurrence of information in a mathematical survey, introduced by Fisher in 1920 (see [9]). If $g$ is positive-definite and all of its components are finite, then $(S, g)$ will be a Riemannian manifold and $g$ is called a Fisher metric on $S$. In this situation, $g$ reads

$$
g_{i j}(\theta)=\int_{\Omega} \partial_{i} p(x ; \theta) \partial_{j} \ell_{\theta} \mathrm{d} x=\int_{\Omega} \frac{1}{p(x ; \theta)} \partial_{i} p(x ; \theta) \partial_{j} p(x ; \theta) \mathrm{d} x .
$$

These kinds of metrics were first studied by Rao in 1945 (see [16]). In a Hausdorff manifold (a customary requirement nowadays), a Riemannian metric induces a distance metric. When the metric is a Fisher one, this distance function (named as Rao distance as well) is an indicator to measure the distance between probability distributions. One can see [15] an implementation of this distance in negative multinomial distributions. For any $\alpha \in \mathbb{R}$, Amari's $\alpha$-connection $\nabla^{\alpha}$ with respect to $p(x ; \theta)$ is defined by the Christoffel symbols

$$
\Gamma_{i j, k}^{(\alpha)}=g\left(\nabla_{\partial_{i}}^{\alpha} \partial_{j}, \partial_{k}\right):=E_{p}\left[\left(\partial_{i} \partial_{j} \ell_{\theta}+\frac{1-\alpha}{2} \partial_{i} \ell_{\theta} \partial_{j} \ell_{\theta}\right)\left(\partial_{k} \ell_{\theta}\right)\right] .
$$

$\alpha$-connections in the case of finite and discrete sample spaces were first studied by Chentsov in 1972 (see the English translation [8]), where is provided a detailed interpretation of the geodesic concept of info-manifolds and of learning curves. Chentsov proved that the Fisher information metric is the unique intrinsic one on the space of probability distributions on a sample space containing at least three points (see also [5] for a historical covering). Later on, Amari studied them in an independent manner and developed the general case by the formula (1.2) in 1982 (see e.g. [2]).

A statistical manifold is a triple $(M, g, \nabla)$, where the manifold $M$ is equipped with a statistical structure $(g, \nabla)$ containing a Riemannian metric $g$ and an affine connection $\nabla$ on $M$, such that the covariant derivative $\nabla g$ is symmetric. A first example appears when $\stackrel{g}{\nabla}$ is the Levi-Civita connection of $g$; this case is a trivial statistical manifold. The motivation behind this definition arises from information geometry, and was first introduced by Lauritzen in 1987 (see [13]). Indeed, one can check that $\nabla^{\alpha}$ is torsion-free and that $\nabla^{\alpha} g$ is totally symmetric, and hence $\left(S, g, \nabla^{\alpha}\right)$ is a statistical manifold. However, dealing with statistical manifolds instead of Fisher metrics is more practical in certain cases. The difference between two $\alpha$ and $\beta$-connections $\nabla^{\alpha}$ and $\nabla^{\beta}$ is given by

$$
\Gamma_{i j, k}^{(\alpha)}-\Gamma_{i j, k}^{(\beta)}=\frac{\beta-\alpha}{2} \mathbb{T}_{i j k}
$$

where $\mathbb{T}$ is a covariant symmetric tensor of degree 3 , defined by $\mathbb{T}_{i j k}:=E_{\theta}\left[\partial_{i} \ell_{\theta} \partial_{j} \ell_{\theta} \partial_{k} \ell_{\theta}\right]$, and is completely identified by its torsion tensor in differential geometry. For the case when $\beta=0,(1.3)$ reduces to

$$
\Gamma_{i j, k}^{(\alpha)}-\stackrel{g}{\Gamma_{i j, k}}=-\frac{\alpha}{2} \mathbb{T}_{i j k}
$$


where $\stackrel{g}{\Gamma}$ ij,k 's are the Christoffel symbols of the Levi-Civita connection induced by $g$. There is a one to one correspondence between the tensors $\mathbb{T}_{i j k}$ and the statistical structures (for a detailed discussion, see [13]).

In differential geometry, the tangent bundle plays an important role. Indeed, this is the first vector bundle over the base manifold and it is very important to geometers in studying the relation between objects on the base manifold and their various kinds of lifts (when such lifts exist) to the tangent bundle. There exists only little research on the lift over the tangent bundle of statistical manifolds (e.g. [12,14]). In [12] the author, by considering a statistical manifold $(M, g, \nabla)$ with skewness operator $K$, introduced a linear connection over $T M$ by using the horizontal lift and the Sasaki lift with respect to the LeviCivita connection of $(M, g)$, and then he studied the conditions that $T M$ together with the Sasaki metric and this connection is a statistical manifold. Also, in [14], the authors focused on the semi-Riemannian Norden metric. Among their results, they showed that $\left(\mathrm{T} M, g^{h}, J\right)$ is an almost complex manifold, where $g^{h}(J \tilde{X}, J \tilde{Y})=-g^{h}(\tilde{X}, \tilde{Y})$, and $g^{h}$ is the horizontal lift of the metric $g$ on $M$ for which $(M, g, \nabla)$ is a statistical manifold, and $J$ is constructed using $\nabla$ in a natural way. In our present work, we investigate the relation between statistical manifolds $(M, g, \nabla)$, and $(\mathrm{T} M, G, \bar{\nabla})$, where $G$ is the Sasaki lift of $g$, and derive several equivalencies for Kähler and holomorphic structures on the tangent bundle.

This paper is organized as follows. Section 2 contains reminders on splitting TT $M$ into the direct sum of its vertical and some horizontal complement. Moreover, we provide a review on the Sasaki lift of a Riemannian metric and the definition of the statistical manifold. In Section 3, we study the statistical manifold (T $M, G, \bar{\nabla})$ induced by a statistical manifold $(M, g, \nabla)$. We find the relation between Christoffel symbols of $\nabla$, and $\bar{\nabla}$. The curvature components of $\bar{\nabla}$ are computed as well. Section 4 is devoted to the holomorphic structures on the tangent space. We derive equivalent conditions for (TM, $G, \bar{J})$ to be Kähler, and some equivalent conditions for (TM, $G, \bar{J}, \bar{\nabla})$ to be holomorphic, where $(M, J, g)$ is a Kähler manifold and where an almost complex structure $\bar{J}$ arises from $J$. We also cover these sections by examples for experimental applications.

\section{Preliminaries}

Let $(M, g)$ be a Riemannian manifold with the unique Levi-Civita connection $\stackrel{g}{\nabla}$. By considering the tangent bundle (TM, $\pi, M)$, we denote as

$$
\mathcal{V}_{(x, y)}=\operatorname{Ker}\left(\mathrm{d} \pi_{(x, y)}\right),
$$

the vertical subspace of $\mathrm{T}_{(x, y)} \mathrm{T} M$ at the point $(x, y)$. Indeed, $\nu_{(x, y)}=\mathrm{T}_{(x, y)} \mathrm{T}_{x} M$. A horizontal subspace is any choice of $\mathcal{H}$ such that

$$
\mathrm{T}_{(x, y)} \mathrm{T} M=\mathcal{H}_{(x, y)} \oplus \mathcal{V}_{(x, y)},
$$

holds. Since $\mathcal{V}=\operatorname{Ker}(\mathrm{d} \pi)$, we infer that $\mathrm{d} \pi: \mathcal{H}_{(x, y)} \longrightarrow \mathrm{T}_{x} M$ is a vector space isomorphism. The fiber $\mathcal{H}_{(x, y)}$ is called the horizontal subspace to T $M$ at $(x, y)$. If the splitting (2.1) holds, then the horizontal lift of a tangent vector $X_{x} \in \mathrm{T}_{x} M$ is the unique vector $X_{x}^{h} \in \mathcal{H}_{(x, y)}$, such that $\mathrm{d} \pi\left(X_{x}^{h}\right)=X_{x}$, and its vertical lift is the unique field $X_{x}^{v} \in \mathcal{V}_{(x, y)}$ which satisfies $X_{x}^{v}(\mathrm{~d} f)=X_{x}(f)$, for all $f \in C^{\infty}(M)$.

Let $(x, \mathcal{U})$ be a local chart on $M$, denoted by its coordinates $x=\left(x^{1}, \cdots, x^{n}\right)$, where $x^{i}$, s belong to $C^{\infty}(M)$. If denote $x^{i} \circ \pi$ by $x^{i}$, and by defining

$$
y^{i}(X)=X\left(x^{i}\right)=\mathrm{d} x^{i}(X), \quad i \in\{1, \cdots, n\}, \quad X \in \mathfrak{X}(M),
$$


one can provide a local chart $\left(x^{1}, \cdots, x^{n}, y^{1}, \cdots, y^{n}\right): \pi^{-1}(\mathcal{U}) \longrightarrow \mathbb{R}^{2 n}$ on TM. Moreover, it can be verified that if $X=X^{i} \frac{\partial}{\partial x^{i}}$, then

$$
X^{v}=X^{i} \frac{\partial}{\partial y^{i}}, \quad X^{h}=X^{i} \frac{\partial}{\partial x^{i}}-X^{j} y^{k} \stackrel{g}{\Gamma_{j k}^{i}} \frac{\partial}{\partial y^{i}},
$$

where $\stackrel{g}{\Gamma_{j k}^{i}}$ are the Christoffel symbols of the Levi-Civita connection $\stackrel{g}{\nabla}$. If $\stackrel{g}{R}$ denotes the Riemann curvature tensor of $\stackrel{g}{\nabla}$, then

$$
\left[X^{v}, Y^{v}\right]=0, \quad\left[X^{h}, Y^{v}\right]=(\stackrel{g}{\nabla} X Y)^{v}, \quad\left[X^{h}, Y^{h}\right]=[X, Y]^{h}-(\stackrel{g}{R}(X, Y) y)^{v},
$$

for any $X, Y, Z \in \mathfrak{X}(M)$ and for any point $(x, y) \in \mathrm{T} M$. The Sasaki metric $G$ on the tangent bundle $\mathrm{T} M$ is a natural lift of the metric $g$ given by

$$
G\left(X^{h}, Y^{h}\right)_{(x, y)}=g_{x}(X, Y), G\left(X^{v}, Y^{h}\right)_{(x, y)}=0, G\left(X^{v}, Y^{v}\right)_{(x, y)}=g_{x}(X, Y) .
$$

If $\left\{\left.\frac{\partial}{\partial x^{i}}\right|_{(x, y)},\left.\frac{\partial}{\partial y^{i}}\right|_{(x, y)}\right\}_{i=1}^{n}$ is the natural basis of $\mathrm{T}_{(x, y)} \mathrm{T} M$, then $\mathcal{H}_{(x, y)}$ can be spanned by $\left\{\left.\frac{\delta}{\delta x^{2}}\right|_{(x, y)}\right\}_{i=1}^{n}$, where

$$
\left.\frac{\delta}{\delta x^{i}}\right|_{(x, y)}=\left.\frac{\partial}{\partial x^{i}}\right|_{(x, y)}-\left.y^{k} \stackrel{g}{\Gamma_{k i}^{j}}(x) \frac{\partial}{\partial y^{j}}\right|_{(x, y)} .
$$

So, its dual basis is $\left\{\mathrm{d} x^{i}, \delta y^{i}\right\}_{i=1}^{n}$, where

$$
\delta y^{i}=\mathrm{d} y^{i}+y^{k} \stackrel{g}{\Gamma_{k j}^{i}}(x) \mathrm{d} x^{j} .
$$

Remark 2.1. In order to simplify the notations, we further write $\partial_{i}, \delta_{i}$ and $\partial_{\bar{i}}$ instead of $\frac{\partial}{\partial x^{i}}, \frac{\delta}{\delta x^{i}}$ and $\frac{\partial}{\partial y^{i}}$, respectively.

The equalities (2.2) and (2.3) have the local expressions

$$
\left[\partial_{\bar{i}}, \partial_{\bar{j}}\right]=0, \quad\left[\delta_{i}, \partial_{\bar{j}}\right] \stackrel{g}{=} \stackrel{\Gamma}{j i}^{k} \partial_{\bar{k}}, \quad\left[\delta_{i}, \delta_{j}\right]=-y^{r} \stackrel{g}{R_{i j r}^{k}} \partial_{\bar{k}}
$$

and

$$
G\left(\frac{\delta}{\delta x^{i}}, \frac{\delta}{\delta x^{j}}\right)_{(x, y)}=g_{\left.i j\right|_{x}}, G\left(\frac{\delta}{\delta x^{i}}, \frac{\partial}{\partial y^{j}}\right)_{(x, y)}=0, G\left(\frac{\partial}{\partial y^{i}}, \frac{\partial}{\partial y^{j}}\right)_{(x, y)}=g_{\left.i j\right|_{x}} .
$$

where the Sasaki metric has the generic form

$$
G_{(x, y)}=g_{i j}(x, y) \mathrm{d} x^{i} \otimes \mathrm{d} x^{j}+g_{i j}(x, y) \delta y^{i} \otimes \delta y^{j} .
$$

It is known that the Levi-Civita connection of the Sasaki metric $G$ is given by [18]:

$$
\left\{\begin{array}{l}
\stackrel{g}{\nabla}_{\delta_{i}} \delta_{j}=\stackrel{\Gamma}{i j}^{k} \delta_{k}-\frac{1}{2} y^{l} \stackrel{g}{R_{i j l}^{k}} \partial_{\bar{k}}, \\
\underline{\nabla}_{\delta_{i}} \partial_{\bar{j}}=\frac{1}{2} y^{l} \stackrel{g}{R_{l j i}^{k} \delta_{k}+\Gamma_{i j}^{k} \partial_{\bar{k}},} \\
\underline{\underline{\nabla}}_{\partial_{\bar{i}}} \delta_{j}=\frac{1}{2} y^{l} \stackrel{g}{R_{l i j}^{k}} \delta_{k}, \quad \stackrel{\underline{g}}{\nabla}_{\partial_{\bar{i}}} \partial_{\bar{j}}=0 .
\end{array}\right.
$$

\subsection{Statistical manifolds}

A statistical manifold is a triple $(M, g, \nabla)$, where $g$ is a Riemannian metric on the manifold $M$, and $\nabla$ is a symmetric linear connection such that the cubic tensor field $\mathrm{C}=\nabla g$ is totally symmetric, namely, the Codazzi equations hold:

$$
\left(\nabla_{X} g\right)(Y, Z)=\left(\nabla_{Y} g\right)(Z, X) \quad\left(=\left(\nabla_{Z} g\right)(X, Y)\right), \forall X, Y, Z \in \mathfrak{X}(M) .
$$

In local coordinates, $\mathcal{C}$ has the following form

$$
\mathcal{C}\left(\partial_{i}, \partial_{j}, \partial_{k}\right)=\partial_{i} g\left(\partial_{j}, \partial_{k}\right)-g\left(\nabla_{\partial_{i}} \partial_{j}, \partial_{k}\right)-g\left(\partial_{j}, \nabla_{\partial_{i}} \partial_{k}\right),
$$


and so

$$
\mathcal{C}_{i j k}=\partial_{k}\left(g_{i j}\right)-\Gamma_{i k}^{h} g_{j h}-\Gamma_{j k}^{h} g_{i h}, \quad \mathcal{C}_{i j k}=\mathcal{C}_{j k i}=\mathcal{C}_{k i j},
$$

where $\Gamma_{j k}^{i}$ are the Christoffel symbols of $\nabla$. Thus, for every statistical manifold $(M, g, \nabla)$, there exists a naturally associated totally symmetric covariant tensor field $\mathcal{C}$ of degree 3. Conversely, let $(M, g, \mathcal{C})$ be a semi-Riemannian manifold with a totally symmetric covariant tensor field $\mathcal{C}$ of degree 3 . If we define the tensor field $A$ by

$$
g(A(X) Y, Z)=\mathcal{C}(X, Y, Z),
$$

and a linear connection $\nabla$ by $\nabla=\stackrel{g}{\nabla}-\frac{A}{2}$, then $\nabla$ is torsion-free and satisfies $\nabla g=\mathcal{C}$. Hence the triple $(M, g, \nabla)$ becomes a statistical manifold. Thus, to equip a statistical structure $(g, \nabla)$ is equivalent to providing a pair of structures $(g, \mathcal{C})$, consisting of a semiRiemannian metric $g$ and a totally symmetric trilinear form $\mathcal{C}$.

For a statistical structure $(\nabla, g)$ we define the difference tensor field $K:=K^{(\nabla, g)} \in \Gamma\left(\mathrm{T} M^{(1,2)}\right)$ as

$$
K(X, Y)=\nabla_{X} Y-\stackrel{g}{\nabla}_{X} Y .
$$

It is easy to check that $K$ is symmetric and, moreover, that we have

$$
g(K(X, Y), Z)=g(Y, K(X, Z)) .
$$

Conversely, if there exists a symmetric tensor field $K \in \Gamma\left(\mathrm{T} M^{(1,2)}\right)$ on a Riemannian manifold $(M, g)$ such that it satisfies the above equation, then $(\nabla=\stackrel{g}{\nabla}+K, g)$ becomes a statistical structure on $M$ (see $[10,11]$ for details).

It is remarkable that considering $K=-\frac{A}{2}$, the two approaches from above, coincide.

\section{Statistical structures on the tangent bundle}

In this section we investigate the condition $(\mathrm{T} M, G, \bar{\nabla})$ to be statistical. Here $G$ is the Sasaki metric of the tangent bundle TM explained in Section 2.

Let $\bar{\nabla}$ be a linear connection on TM. Then, with respect to $\left\{\delta_{i}, \partial_{\bar{i}}\right\}$, we have:

$$
\begin{array}{ll}
\bar{\nabla}_{\delta_{i}} \delta_{j}=\bar{\Gamma}_{i j}^{k} \delta_{k}+\bar{\Gamma}_{i j}^{\bar{k}} \partial_{\bar{k}}, & \bar{\nabla}_{\delta_{i}} \partial_{\bar{j}}=\bar{\Gamma}_{i \bar{j}}^{k} \delta_{k}+\bar{\Gamma}_{i \bar{j}}^{\bar{k}} \partial_{\bar{k}}, \\
\bar{\nabla}_{\partial_{\bar{i}}} \delta_{j}=\bar{\Gamma}_{\bar{i} j}^{k} \delta_{k}+\bar{\Gamma}_{\bar{i} j}^{\bar{k}} \partial_{\bar{k}}, & \bar{\nabla}_{\partial_{\bar{i}}} \partial_{\bar{j}}=\bar{\Gamma}_{\overline{i j}}^{k} \delta_{k}+\bar{\Gamma}_{\bar{i} j}^{\bar{k}} \partial_{\bar{k}},
\end{array}
$$

where $\bar{\Gamma}_{A B}^{C}, A, B, C \in\{1, \cdots, n, \overline{1}, \cdots, \bar{n}\}$ are smooth functions on TM. By using the torsion-freeness conditions, we can get the equivalent local conditions as follows.

Lemma 3.1. The symmetry of $\bar{\nabla}$ has the following local alternative form:
i) $\bar{\Gamma}_{i \bar{j}}^{k}=\bar{\Gamma}_{\bar{j} i}^{k}$,
ii) $\bar{\Gamma}_{i \bar{j}}^{\bar{k}}-\bar{\Gamma} \bar{j}_{\bar{j} i}^{\bar{k}}=\stackrel{g}{\Gamma_{i j}^{k}}$,
iii) $\bar{\Gamma}_{i j}^{k}=\bar{\Gamma}_{j i}^{k}$,
iv) $\bar{\Gamma}_{i j}^{\bar{k}}-\bar{\Gamma}_{j i}^{\bar{k}}=-y^{r} R_{i j r}^{k}$,
v) $\bar{\Gamma} \frac{k}{i j}=\bar{\Gamma} \frac{k}{j i}$,
vi) $\bar{\Gamma} \bar{i} \bar{k}=\bar{\Gamma} \bar{j} \bar{k}$.

Proof. The equations from the first line of the Lemma, are obtained by

$$
\bar{\nabla}_{\delta_{j}} \partial_{\bar{i}}-\bar{\nabla}_{\partial_{\bar{i}}} \delta_{j}=\left[\delta_{j}, \partial_{\bar{i}}\right] \stackrel{g}{=} \Gamma_{j i}^{k} \partial_{\bar{k}} .
$$

The equations from the second line, can be verified by

$$
\bar{\nabla}_{\delta_{i}} \delta_{j}-\bar{\nabla}_{\delta_{j}} \delta_{i}=\left[\delta_{i}, \delta_{j}\right]=-y^{r} \stackrel{g}{R_{i j r}^{k}} \partial_{\bar{k}},
$$

and the ones from the third line result as $\bar{\nabla}_{\partial_{\bar{i}}} \partial_{\bar{j}}-\bar{\nabla}_{\partial_{\bar{j}}} \partial_{\bar{i}}=\left[\partial_{\bar{i}}, \partial_{\bar{j}}\right]=0$. 
Lemma 3.2. Let $(M, g, \nabla)$ be a statistical manifold. Then, applying the Codazzi equations for the statistical manifold $(\mathrm{T} M, G, \bar{\nabla})$, we yield

$$
\begin{aligned}
& \text { i) } \partial_{i} g_{j k}-\bar{\Gamma}_{i j}^{r} g_{r k}-\bar{\Gamma}_{i k}^{r} g_{r j}=\partial_{j} g_{k i}-\bar{\Gamma}_{j k}^{r} g_{r i}-\bar{\Gamma}_{j i}^{r} g_{r k}=\partial_{k} g_{i j}-\bar{\Gamma}_{k i}^{r} g_{r j}-\bar{\Gamma}_{k j}^{r} g_{r i}, \\
& \text { ii) } \bar{\Gamma}_{i j}^{\bar{r}} g_{r k}+\bar{\Gamma}_{i \bar{k}}^{r} g_{j r}=\bar{\Gamma}_{j \bar{k}}^{r} g_{r i}+\bar{\Gamma}_{j i}^{\bar{r}} g_{k r}=\bar{\Gamma}_{\bar{k} i}^{r} g_{r j}+\bar{\Gamma}_{\bar{k} j}^{r} g_{r i}, \\
& \text { iii) } \partial_{i} g_{j k}-\bar{\Gamma}_{i \bar{j}}^{\bar{r}} g_{r k}-\bar{\Gamma}_{i \bar{k}}^{\bar{r}} g_{j r}=-\bar{\Gamma}_{\bar{j} \bar{k}}^{r} g_{r i}-\bar{\Gamma}_{\bar{j}}^{\bar{r}} g_{r k}=-\bar{\Gamma}_{\bar{k} i}^{\bar{r}} g_{r j}-\bar{\Gamma}_{\bar{k} \bar{j}}^{r} g_{i r}, \\
& \text { iv) } \bar{\Gamma}_{\bar{i} \bar{j}}^{\bar{r}} g_{r k}+\bar{\Gamma}_{\bar{i} \bar{k}}^{\bar{r}} g_{r j}=\bar{\Gamma}_{\bar{j} \bar{k}}^{\bar{r}} g_{r i}+\bar{\Gamma}_{\bar{j} \bar{r}}^{\bar{r}} g_{r k}=\bar{\Gamma}_{\bar{k} \bar{r}}^{\bar{r}} g_{r j}+\bar{\Gamma}_{\bar{k} \bar{j}}^{\bar{r}} g_{r i} .
\end{aligned}
$$

Proof. By using

$$
\left(\bar{\nabla}_{\delta_{i}} G\right)\left(\delta_{j}, \delta_{k}\right)=\partial_{i} g_{j k}-\bar{\Gamma}_{i j}^{r} g_{r k}-\bar{\Gamma}_{i k}^{r} g_{r j},
$$

and the Codazzi equation

$$
\left(\bar{\nabla}_{\delta_{i}} G\right)\left(\delta_{j}, \delta_{k}\right)=\left(\bar{\nabla}_{\delta_{j}} G\right)\left(\delta_{k}, \delta_{i}\right)=\left(\bar{\nabla}_{\delta_{k}} G\right)\left(\delta_{i}, \delta_{j}\right)
$$

we get (3.1). Also, the equations

$$
\left\{\begin{array}{l}
\left(\bar{\nabla}_{\delta_{i}} G\right)\left(\delta_{j}, \partial_{\bar{k}}\right)=-\bar{\Gamma}_{i j}^{\bar{r}} g_{r k}-\bar{\Gamma}_{i \bar{k}}^{r} g_{j r}, \\
\left(\bar{\nabla}_{\delta_{j}} G\right)\left(\partial_{\bar{k}}, \delta_{i}\right)=-\bar{\Gamma}_{j \bar{k}}^{\bar{r}} g_{r i}-\bar{\Gamma}_{j i}^{r} g_{k r}, \\
\left(\bar{\nabla}_{\partial_{\bar{k}}} G\right)\left(\delta_{i}, \delta_{j}\right)=-\bar{\Gamma}_{\bar{k} i}^{r} g_{r j}-\bar{\Gamma}_{\bar{k} j}^{r} g_{r i}
\end{array}\right.
$$

and the Codazzi equations

$$
\left(\bar{\nabla}_{\delta_{i}} G\right)\left(\delta_{j}, \partial_{\bar{k}}\right)=\left(\bar{\nabla}_{\delta_{j}} G\right)\left(\partial_{\bar{k}}, \delta_{i}\right)=\left(\bar{\nabla}_{\partial_{\bar{k}}} G\right)\left(\delta_{i}, \delta_{j}\right),
$$

imply (3.2). Similarly, from

$$
\left\{\begin{array}{l}
\left(\bar{\nabla}_{\delta_{i}} G\right)\left(\partial_{\bar{j}}, \partial_{\bar{k}}\right)=\partial_{i} g_{j k}-\bar{\Gamma}_{i \bar{j}}^{\bar{r}} g_{r k}-\bar{\Gamma}_{i \bar{k}}^{\bar{r}} g_{j r} \\
\left(\bar{\nabla}_{\partial_{\bar{j}}} G\right)\left(\partial_{\bar{k}}, \delta_{i}\right)=-\bar{\Gamma}_{\bar{j} \bar{k}}^{r} g_{r i}-\bar{\Gamma}_{\bar{j} i}^{\bar{r}} g_{r k} \\
\left(\bar{\nabla}_{\partial_{\bar{k}}} G\right)\left(\delta_{i}, \partial_{\bar{j}}\right)=-\bar{\Gamma}_{\bar{k} i}^{\bar{r}} g_{r j}-\bar{\Gamma}_{\bar{k} \bar{j}}^{r} g_{i r}
\end{array}\right.
$$

and from the Codazzi equations

$$
\left(\bar{\nabla}_{\delta_{i}} G\right)\left(\partial_{\bar{j}}, \partial_{\bar{k}}\right)=\left(\bar{\nabla}_{\partial_{j}} G\right)\left(\partial_{\bar{k}}, \delta_{i}\right)=\left(\bar{\nabla}_{\partial_{\bar{k}}} G\right)\left(\delta_{i}, \partial_{\bar{j}}\right),
$$

we get (3.3). Finally, from

$$
\left(\bar{\nabla}_{\partial_{\bar{i}}} G\right)\left(\partial_{\bar{j}}, \partial_{\bar{k}}\right)=-\bar{\Gamma}_{\bar{i} j}^{\bar{r}} g_{r k}-\bar{\Gamma}_{\bar{i} \bar{k}}^{\bar{r}} g_{r j}
$$

and from the Codazzi equations

$$
\left(\bar{\nabla}_{\partial_{\bar{i}}} G\right)\left(\partial_{\bar{j}}, \partial_{\bar{k}}\right)=\left(\bar{\nabla}_{\partial_{\bar{j}}} G\right)\left(\partial_{\bar{k}}, \partial_{\bar{i}}\right)=\left(\bar{\nabla}_{\partial_{\bar{k}}} G\right)\left(\partial_{\bar{i}}, \partial_{\bar{j}}\right),
$$

we infer (3.4).

Proposition 3.3. Let $(M, g, \nabla)$ be a statistical manifold. Then $(\mathrm{T} M, G, \bar{\nabla})$ is a statistical manifold if and only if

$$
\begin{aligned}
& \left(\bar{\Gamma}_{i k}^{r}-\Gamma_{i k}^{r}\right) g_{r j}=\left(\bar{\Gamma}_{j k}^{r}-\Gamma_{j k}^{r}\right) g_{r i}, \\
& \bar{\Gamma}_{i j}^{\bar{r}} g_{r k}=\bar{\Gamma}_{\bar{k} j}^{r} g_{r i}, \\
& \bar{\Gamma}_{i \bar{k}}^{r} g_{j r}-y^{m} \stackrel{g}{R}_{i j m k}=\bar{\Gamma}_{j \bar{k}}^{r} g_{r i}, \\
& \left(\bar{\Gamma}_{i \bar{k}}^{\bar{r}}-\stackrel{\Gamma}{ }_{i k}^{r}\right) g_{j r}=\bar{\Gamma}_{\bar{j} \bar{k}}^{r} g_{r i}, \\
& \bar{\Gamma}^{\bar{r}}{ }_{j}^{\bar{r}} g_{r k}=\bar{\Gamma}_{\bar{k} i}^{\bar{r}} g_{r j}, \\
& \bar{\Gamma}_{\bar{i} \bar{k}}^{\bar{r}} g_{r j}=\bar{\Gamma}_{\bar{j} \bar{k}}^{\bar{r}} g_{r i},
\end{aligned}
$$

where $\stackrel{g}{R}_{i j m k}=\stackrel{g}{R}$ ijm $g_{r k}$. 
Proof. From (i) of Lemma 3.2, we obtain

$$
\partial_{i} g_{j k}-\partial_{j} g_{k i}=\bar{\Gamma}_{i k}^{r} g_{r j}-\bar{\Gamma}_{j k}^{r} g_{r i} .
$$

On the other hand, by applying the Codazzi equations for the couple of $g$ and $\nabla$, we get

$$
\partial_{i} g_{j k}-\partial_{j} g_{k i}=\Gamma_{i k}^{r} g_{r j}-\Gamma_{j k}^{r} g_{r i} .
$$

From (3.11) and (3.12), we have the first claimed equation (3.5). From the first equality of (ii) in Lemma 3.2 and (iv) of Lemma 3.1, we get (3.7). From the equality of the first and the last sides of (ii) of Lemma 3.2 and (i) of Lemma 3.1, we get (3.6). We note that (3.6) could be obtained from the last equality of (ii) of Lemma 3.2. From (ii) in Lemma 3.1 and the first equality of (iii) in Lemma 3.2, we obtain

$$
\partial_{i} g_{j k}-\stackrel{g}{\Gamma}{ }_{i j}^{r} g_{r k}=\bar{\Gamma}_{i \bar{k}}^{\bar{r}} g_{j r}-\bar{\Gamma}_{\bar{j} \bar{k}}^{r} g_{r i} .
$$

But, from the compatibility condition of the Levi-Civita connection $\stackrel{g}{\nabla}$ with the metric $g$, we will have

$$
\partial_{i} g_{j k}=\stackrel{g}{\Gamma}{ }_{i j}^{r} g_{r k}+\stackrel{g}{\Gamma}{ }_{i k}^{r} g_{j r} .
$$

From (3.13) and (3.14), we obtain (3.8). We note that the first and the third sides of (iii) of Lemma 3.2 could be applied to infer the same result. From the last equation of (iii) of Lemma 3.2 and (v) of Lemma 3.1, we get (3.9). As well, from (iv) of Lemma 3.2 and (vi) of Lemma (3.1), we get (3.10).

Example 3.4. Let $(M, g, \nabla)$ be a statistical manifold and let $\mathcal{C}$ be the cubic tensor field of $\nabla$, with the coefficients

$$
\mathcal{C}_{i j k}=\partial_{k} g_{i j}-\Gamma_{i k}^{h} g_{j h}-\Gamma_{j k}^{h} g_{i h} .
$$

Now we consider the horizontal lift $\mathcal{C}^{h}$ of $\mathcal{C}$ (see $[18$, p. 97]). Then we have

$$
\left\{\begin{aligned}
& \mathrm{C}^{h}\left(\delta_{i}, \delta_{j}, \partial_{\bar{k}}\right)=\mathrm{C}^{h}\left(\delta_{i}, \partial_{\bar{j}}, \delta_{k}\right)=\mathrm{C}^{h}\left(\delta_{i}, \partial_{\bar{j}}, \delta_{k}\right)=\left(\mathfrak{C}\left(\partial_{i}, \partial_{j}, \partial_{k}\right)\right)^{v}=\left(\mathcal{C}_{i j k}\right)^{v}=\mathcal{C}_{i j k} \circ \pi, \\
& \mathrm{C}^{h}\left(\delta_{i}, \delta_{j}, \delta_{k}\right)=\left(\mathcal{C}\left(\partial_{i}, \partial_{j}, \partial_{k}\right)\right)^{h}=\left(\mathcal{C}_{i j k}\right)^{h}=0, \\
& \mathrm{C}^{h}\left(\partial_{\bar{i}}, \partial_{\bar{j}}, \partial_{\bar{k}}\right)=0 .
\end{aligned}\right.
$$

Since $\mathrm{e}^{h}$ is symmetric, then using (2.6) we infer that there exists the tensor field $\tilde{A}$ such that

$$
G(\tilde{A}(\tilde{X}) \tilde{Y}, \tilde{Z})=\mathcal{C}^{h}(\tilde{X}, \tilde{Y}, \tilde{Z}) .
$$

By setting $\tilde{X}=\delta_{i}, \tilde{Y}=\delta_{j}$ and $\tilde{Z}=\delta_{r}$ in (3.15) we deduce that the horizontal part of $\tilde{A}\left(\delta_{i}\right) \delta_{j}$ is zero. Similarly, setting $\tilde{X}=\delta_{i}, \tilde{Y}=\delta_{j}$ and $\tilde{Z}=\partial_{\bar{r}}$ in (3.15) we deduce that the vertical part of $\tilde{A}\left(\delta_{i}\right) \delta_{j}$ is $g^{k r} \mathrm{C}_{i j r} \partial_{\bar{k}}$. So we have

$$
\tilde{A}\left(\delta_{i}\right) \delta_{j}=g^{k r} \mathcal{C}_{i j r} \partial_{\bar{k}}
$$

In a similar way we can obtain the following equations:

$$
\begin{aligned}
& \tilde{A}\left(\delta_{i}\right) \partial_{\bar{j}}=g^{k r} \mathcal{C}_{i j r} \delta_{k}+g^{k r} \mathcal{C}_{i j r} \partial_{\bar{k}}, \\
& \tilde{A}\left(\partial_{\bar{i}}\right) \delta_{j}=g^{k r} \mathcal{C}_{j i r} \delta_{k}+g^{k r} \mathcal{C}_{j i r} \partial_{\bar{k}}, \\
& \tilde{A}\left(\partial_{\bar{i}}\right) \partial_{\bar{j}}=g^{k r} \mathcal{C}_{i j r} \delta_{k} .
\end{aligned}
$$


Now we consider the Levi-Civita connection $\stackrel{g}{\nabla}$ of Sasaki metric $G$ and the linear connection $\bar{\nabla}=\stackrel{g}{\nabla}-\frac{\tilde{A}}{2}$. Then using $(2.5)$ and $(3.16)-(3.17)$ we get

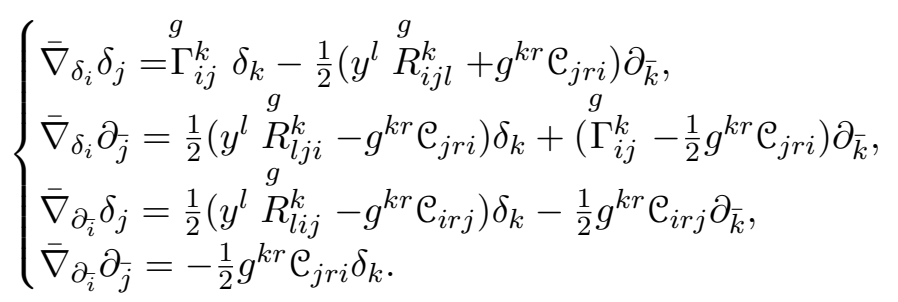

From the first equation of (3.18) we have

$$
\left(\bar{\Gamma}_{i k}^{r}-\Gamma_{i k}^{r}\right) g_{r j}=\left(\bar{\Gamma}_{i k}^{r}-\Gamma_{i k}^{r}\right) g_{r j} .
$$

Since $K\left(\partial_{i}, \partial_{j}\right)=\left(\Gamma_{i j}^{r}-\stackrel{g}{\Gamma_{i j}^{r}}\right) \partial_{r}$, then using $(2.7)$ we get

$$
\left(\stackrel{g}{\Gamma}_{i k}^{r}-\Gamma_{i k}^{r}\right) g_{r j}=\left(\stackrel{g}{\Gamma_{k j}^{r}}-\Gamma_{k j}^{r}\right) g_{i r} .
$$

Finally, (3.19) and (3.20) imply

$$
\left(\bar{\Gamma}_{i k}^{r}-\Gamma_{i k}^{r}\right) g_{r j}=\left(\bar{\Gamma}_{k j}^{r}-\Gamma_{k j}^{r}\right) g_{i r}=\left(\bar{\Gamma}_{k j}^{r}-\Gamma_{k j}^{r}\right) g_{i r}
$$

Thus the Christoffel coefficients of $\bar{\nabla}$ satisfy (3.5). Similarly, we note that the other equations of Proposition 3.3 hold true for $\bar{\nabla}$, and $(G, \bar{\nabla})$ is a statistical structure on TM.

Remark 3.5. The statistical structure introduced by Example 3.4 has been studied by Matsuzoe-Inoguchi in [14]. They globally showed that if $(M, g, \nabla)$ is a statistical manifold, then $\left(T M, G, C^{h}\right)$ is a statistical one too.

By using the definition of the curvature tensor of $\bar{\nabla}$, i.e.,

$$
\bar{R}(\tilde{X}, \tilde{Y}) \tilde{Z}=\bar{\nabla}_{\tilde{X}} \bar{\nabla}_{\tilde{Y}} \tilde{Z}-\bar{\nabla}_{\tilde{Y}} \bar{\nabla}_{\tilde{X}} \tilde{Z}-\bar{\nabla}_{[\tilde{X}, \tilde{Y}]} \tilde{Z}, \quad \forall \tilde{X}, \tilde{Y}, \tilde{Z} \in X(\mathrm{~T} M),
$$

and by using (2.4) we conclude the following:

Proposition 3.6. Let $(M, g, \nabla)$ be a statistical manifold such that the Riemannian curvature of $(M, g)$ is zero. Then $(\mathrm{T} M, G, \bar{\nabla})$ is flat if and only if

$$
g^{m r} \mathrm{C}_{k r j} \mathfrak{C}_{i l m}=0, \quad \stackrel{g}{\nabla} \mathrm{D}_{\partial_{i}} \mathrm{e}_{j l k}=0,
$$

where $(G, \bar{\nabla})$ is the statistical structure on TM given by Example 3.4 .

Proposition 3.7. Let $\left(\mathbb{E}^{n}, g, \nabla\right)$ be a statistical manifold, where $g_{i j}=\delta_{i j}$ is the Euclidean metric and $\Gamma_{j k}^{i}$ are the Christoffel symbols of $\nabla$. Then $\left(\mathrm{TE}^{n}, G, \bar{\nabla}\right)$ is flat if and only if $\bar{\nabla}$ reduces to the Levi-Civita connection $\stackrel{g}{\nabla}$.

Proof. From the first equation of $(3.21)$ we conclude that $\left(\mathrm{TE}^{n}, G, \bar{\nabla}\right)$ is flat if and only if $\mathcal{C}_{k m j} \mathfrak{C}_{i l m}=0$, where $i, j, k, l, m \in\{1, \cdots, n\}$. But this equation is equivalent with $\mathcal{C}_{i l m}=0$, for all $i, l, m \in\{1, \cdots, n\}$.

Example 3.8. Consider the Fisher metric

$$
g=\left[\begin{array}{cc}
\frac{1}{x_{2}^{2}} & 0 \\
0 & \frac{2}{x_{2}^{2}}
\end{array}\right],
$$


on $M=\left\{\left(x_{1}, x_{2}\right): x_{1} \in \mathbb{R}, x_{2} \in \mathbb{R}_{+}\right\}$of the normal distribution, with the local coordinates $\left(x_{1}, x_{2}\right)$. It is easy to see that

$$
g^{-1}=\left[\begin{array}{cc}
x_{2}^{2} & 0 \\
0 & \frac{x_{2}^{2}}{2}
\end{array}\right]
$$

We choose the trivial statistical structure $(g, \nabla:=\stackrel{g}{\nabla})$ on $M$. So, $\Gamma_{i j}^{k} \stackrel{g}{=} \Gamma_{i j}^{k}$, and we shall have

$$
\Gamma_{11}^{1}=\Gamma_{22}^{1}=\Gamma_{12}^{2}=\Gamma_{21}^{2}=0, \quad \Gamma_{12}^{1}=\Gamma_{21}^{1}=-\frac{1}{x_{2}}, \quad \Gamma_{11}^{2}=\frac{1}{2 x_{2}}, \quad \Gamma_{22}^{2}=-\frac{1}{x_{2}} .
$$

It can be verified that all the Riemannian curvature components of the Fisher metric (3.22) are zero except than the following components:

$$
\stackrel{g}{R_{121}^{2}}=-\stackrel{g}{R}{ }_{211}^{2}=\frac{1}{2 x_{2}^{2}}, \quad \stackrel{g}{R}{ }_{122}^{1}=-\stackrel{g}{R}{ }_{212}^{1}=-\frac{1}{x_{2}^{2}} .
$$

Now, we are going to compute all of the $\bar{\Gamma}_{\text {.. }}$ 's. We use Lemma 3.1 by some technical efforts to this aim and only mention details of one of these computations. Say, applying $i=k=1$ and $j=2$ in (3.5) together with (iii) of Lemma 3.1, leads to

$$
\bar{\Gamma}_{12}^{2}=\bar{\Gamma}_{21}^{2}=2\left(\bar{\Gamma}_{11}^{2}-\frac{1}{x_{2}}\right) .
$$

There are 17 independent Christoffel symbols on which the other depend. These symbols are synthetically presented by the following table.

A synthetic table for the Christoffel symbols of $\bar{\nabla}$ from Example 3.8. 


\begin{tabular}{|c|c|}
\hline$\alpha=\bar{\Gamma}_{11}^{1}$ & - \\
\hline$\beta=\bar{\Gamma}_{11}^{2}$ & $\bar{\Gamma}_{12}^{2}=\bar{\Gamma}_{21}^{2}=2\left(\beta-\frac{1}{x_{2}}\right), \quad \bar{\Gamma}_{12}^{1}=\bar{\Gamma}_{21}^{1}=2 \beta-\frac{1}{x_{2}}, \quad \bar{\Gamma}_{22}^{1}=4\left(\beta-\frac{1}{x_{2}}\right)$ \\
\hline$\gamma=\bar{\Gamma}_{11}^{\overline{1}}$ & $\bar{\Gamma}_{\overline{1} 1}^{1}=\bar{\Gamma}_{1 \overline{1}}^{1}=\gamma$ \\
\hline$\delta=\bar{\Gamma}_{11}^{\overline{2}}$ & $\bar{\Gamma}_{\overline{2} 1}^{1}=\bar{\Gamma}_{1 \overline{2}}^{1}=2 \delta$ \\
\hline$\epsilon=\bar{\Gamma}_{12}^{\overline{1}}$ & $\bar{\Gamma}_{1 \overline{1}}^{2}=\bar{\Gamma}_{\overline{1} 1}^{2}=\frac{1}{2} \epsilon, \quad \bar{\Gamma}_{\overline{1} 2}^{1}=\bar{\Gamma}_{2 \overline{1}}^{1}=\epsilon-\frac{y_{2}}{x_{2}^{2}}, \quad \bar{\Gamma}_{21}^{\overline{1}}=\epsilon-\frac{y_{2}}{x_{2}^{2}}$ \\
\hline$\varepsilon=\bar{\Gamma}_{12}^{\overline{2}}$ & $\bar{\Gamma}_{\overline{2} 2}^{1}=\bar{\Gamma}_{2 \overline{2}}^{1}=2 \varepsilon+\frac{y_{1}}{2 x_{2}^{2}}, \quad 2 \bar{\Gamma}_{21}^{\overline{2}}=2 \varepsilon+\frac{y_{1}}{2 x_{2}^{2}}, \quad \bar{\Gamma}_{\overline{2} 1}^{2}=\bar{\Gamma}_{1 \overline{2}}^{2}=\varepsilon$ \\
\hline$\zeta=\bar{\Gamma}_{22}^{2}$ & - \\
\hline$\eta=\bar{\Gamma}_{22}^{\overline{1}}$ & $\bar{\Gamma}_{\overline{1} 2}^{2}=\bar{\Gamma}_{2 \overline{1}}^{2}=\frac{1}{2} \eta$ \\
\hline$\theta=\bar{\Gamma}_{22}^{\overline{2}}$ & $\bar{\Gamma}_{\overline{2} 2}^{2}=\bar{\Gamma}_{2 \overline{2}}^{2}=\theta$ \\
\hline$\vartheta=\bar{\Gamma}_{\overline{1} 1}^{\overline{1}}$ & $\bar{\Gamma}_{\overline{1} \overline{1}}^{1}=\bar{\Gamma}_{1 \overline{1}}^{\overline{1}}=\vartheta$ \\
\hline$\iota=\bar{\Gamma}_{\overline{1} 2}^{\overline{1}}$ & $\bar{\Gamma}_{\overline{1} \overline{1}}^{2}=\frac{1}{2} \iota, \quad \bar{\Gamma}_{2 \overline{1}}^{\overline{1}}=\iota-\frac{1}{x_{2}}$ \\
\hline$\kappa=\bar{\Gamma}_{\overline{2} \overline{2}}^{1}$ & $\bar{\Gamma}_{\overline{1} 1}^{\overline{2}}=\bar{\Gamma}_{\overline{2} 1}^{\overline{2}}=\bar{\Gamma}_{1 \overline{2}}^{\overline{2}}=\frac{1}{2} \kappa, \quad \bar{\Gamma}_{\overline{2} \overline{1}}^{1}=\bar{\Gamma}_{\overline{1} \overline{2}}^{1}=\bar{\Gamma}_{\overline{2} 1}^{\overline{1}}=\kappa, \quad \bar{\Gamma}_{1 \overline{2}}^{\overline{1}}=\kappa-\frac{1}{x_{2}}, \quad \bar{\Gamma}_{1 \overline{1}}^{\overline{2}}=\frac{1}{2}\left(\kappa+\frac{1}{x_{2}}\right)$ \\
\hline$\lambda=\bar{\Gamma}_{\overline{2} \overline{2}}^{2}$ & $\bar{\Gamma}_{\overline{1} 2}^{\overline{2}}=\bar{\Gamma}_{2 \overline{1}}^{\overline{2}}=\bar{\Gamma}_{\overline{1} \overline{2}}^{2}=\bar{\Gamma}_{\overline{2} \overline{1}}^{2}=\frac{1}{2} \lambda, \quad \bar{\Gamma}_{2 \overline{2}}^{\overline{1}}=\bar{\Gamma}_{\overline{2} 2}^{\overline{1}}=\Gamma_{\overline{2} 2}^{\overline{2}}=\lambda, \quad \bar{\Gamma}_{2 \overline{2}}^{\overline{2}}=\lambda-\frac{1}{x_{2}}$ \\
\hline$\mu=\bar{\Gamma} \overline{\overline{1}} \overline{2}$ & $\bar{\Gamma}_{\overline{1} \overline{2}}^{\overline{2}}=\bar{\Gamma}_{\overline{2} \overline{1}}^{\overline{2}}=\frac{1}{2} \mu$ \\
\hline$\nu=\bar{\Gamma}_{\overline{2}}^{\overline{2}} \overline{2}$ & - \\
\hline$\xi=\bar{\Gamma}_{\overline{1}}^{\overline{1}} \overline{1}$ & - \\
\hline$\pi=\bar{\Gamma}_{\overline{1} \overline{1}}^{\overline{2}}$ & $\bar{\Gamma}_{\overline{1} \overline{2}} \overline{1}=2 \pi, \quad \bar{\Gamma}_{\overline{2}} \overline{1}=2 \pi$ \\
\hline
\end{tabular}

Example 3.9. Consider the $n$-dimensional Euclidean space $\mathbb{E}^{n}$ equipped with the Riemannian metric $g_{i j}=\delta_{i j}$. It is known that all of the Christoffel symbols induced by the Levi-Civita connection $\stackrel{g}{\nabla}$ are zero. So, also $\stackrel{g}{\Gamma_{i j}^{k}}$ are zero and this space is flat. Now, we equip it by the trivial statistical structure $(g, \nabla:=\stackrel{g}{\nabla})$. By plugging in these data in Proposition 3.3 , we can characterize all of the induced statistical structures $(G, \bar{\nabla})$ on $\mathrm{TE}^{n}$, as follows

$$
\bar{\Gamma}_{i k}^{j}=\bar{\Gamma}_{j k}^{i}, \quad \bar{\Gamma}_{i j}^{\bar{k}}=\bar{\Gamma}_{\bar{k} j}^{i}, \quad \bar{\Gamma}_{i \bar{k}}^{j}=\bar{\Gamma}_{j \bar{k}}^{i}, \quad \bar{\Gamma}_{i \bar{k}}^{\bar{j}}=\bar{\Gamma}_{\bar{j} \bar{k}}^{i}, \quad \bar{\Gamma}_{\bar{j} i}^{\bar{k}}=\bar{\Gamma}_{\bar{k} i}^{\bar{j}}, \quad \bar{\Gamma}_{\bar{i} \bar{k}}^{\bar{j}}=\bar{\Gamma}_{\bar{j} \bar{k}}^{\bar{i}}
$$

For instance, the couple $(G, \bar{\nabla})$ is a statistical structure on $\mathrm{TE}^{n}$, where $\bar{\nabla}$ has the coefficients

$$
\left\{\begin{array}{l}
\bar{\Gamma}_{i j}^{k}=f_{1}\left(x_{1}, \cdots, x_{n}, y_{1}, \cdots, y_{n}\right), \\
\bar{\Gamma}_{i j}^{k}=\bar{\Gamma}_{\bar{i} j}^{k}=\bar{\Gamma}_{i \bar{j}}^{k}=f_{2}\left(x_{1}, \cdots, x_{n}, y_{1}, \cdots, y_{n}\right), \\
\bar{\Gamma} \overline{\bar{i}}=\bar{\Gamma}_{i \bar{j}}^{k}=\bar{\Gamma} \overline{\bar{i}} \overline{i j}=f_{3}\left(x_{1}, \cdots, x_{n}, y_{1}, \cdots, y_{n}\right), \\
\bar{\Gamma} \bar{k} \bar{k}=f_{4}\left(x_{1}, \cdots, x_{n}, y_{1}, \cdots, y_{n}\right) .
\end{array}\right.
$$




\section{Holomorphic statistical manifolds}

Let $(M, g)$ be a Riemannian manifold, (T $M, \pi, M)$ be its tangent bundle on a domain $\mathcal{U} \subseteq M$ of a local chart, and let $g$ have the components $g_{i j}(x), \quad(i, j \in\{1, \cdots, n\})$. On $\pi^{-1}(\mathcal{U}) \subseteq \mathrm{T} M$, we consider $\tau=g_{i j}(x) y^{i} y^{j}$. Then $\tau$ is globally defined and differentiable on $\mathrm{T} M$.

A $(1,1)$-tensor field $J$ on a differential manifold $M$ with dimension $n=2 m \geq 2$ is called an almost complex structure if $J^{2}=-\mathrm{Id}$. Also, an almost Hermitian manifold $(M, g, J)$ is defined to be a differential manifold $M$ endowed with an almost complex structure $J$ and a semi-Riemannian metric $g$, such that $g(J X, J Y)=g(X, Y)$, for all $X, Y \in \chi(M)$. Moreover, if the 2-form $\omega(X, Y)=g(X, J Y)$ is closed, then $(M, g, J)$ is called an almost Kähler manifold. In the case when $J$ is integrable, then we have the definition of a Kähler manifold.

Let $(M, g, J)$ be a Kähler manifold. Then the above conditions in local form reduce to the following equations

$$
\left\{\begin{array}{l}
J_{i}^{j} J_{j}^{k}=-\delta_{i}^{k}, \quad J_{i}^{k} J_{j k}=g_{i j}, \quad \omega_{i j}=J_{j i}, \quad \partial_{i} J_{k j}+\partial_{j} J_{i k}+\partial_{k} J_{j i}=0, \\
J_{i}^{m} \partial_{m}\left(J_{j}^{s}\right)-J_{j}^{m} \partial_{m}\left(J_{i}^{s}\right)-\partial_{i}\left(J_{j}^{m}\right) J_{m}^{s}+\partial_{j}\left(J_{i}^{m}\right) J_{m}^{s}=0,
\end{array}\right.
$$

where $J_{j k}=J_{j}^{r} g_{r k}$. Note that two first equations give $J_{j k}=-J_{k j}$.

Now, we define an almost complex structure $\bar{J}$ on the open set $\pi^{-1}(\mathcal{U}) \subseteq \mathrm{T} M$ by

$$
\left\{\begin{array}{l}
\bar{J} \delta_{i}=B \partial_{\bar{i}}+A J_{i}^{j} \partial_{\bar{j}}=\left(B \delta_{i}^{j}+A J_{i}^{j}\right) \partial_{\bar{j}}, \\
\bar{J} \partial_{\bar{i}}=-\frac{B}{A^{2}+B^{2}} \delta_{i}+\frac{A}{A^{2}+B^{2}} J_{i}^{j} \delta_{j}=\left(-\frac{B}{A^{2}+B^{2}} \delta_{i}^{j}+\frac{A}{A^{2}+B^{2}} J_{i}^{j}\right) \delta_{j},
\end{array}\right.
$$

where $A$ and $B$ are functions with respect to $\tau$.

Lemma 4.1. (TM, $G, \bar{J})$ is an almost Hermitian structure if and only if

$$
A^{2}+B^{2}=1 \text {. }
$$

Proof. Direct calculations give us $G\left(\bar{J} \delta_{i}, \bar{J} \partial_{\bar{j}}\right)=0=G\left(\delta_{i}, \partial_{\bar{j}}\right), G\left(\bar{J} \delta_{i}, \bar{J} \partial_{\delta_{j}}\right)=\left(A^{2}+\right.$ $\left.B^{2}\right) G\left(\delta_{i}, \delta_{j}\right), G\left(\bar{J} \partial_{\bar{i}}, \bar{J} \partial_{\bar{j}}\right)=\frac{1}{A^{2}+B^{2}} G\left(\partial_{\bar{i}}, \partial_{\bar{j}}\right)$. Thus, $G(\bar{J} X, \bar{J} Y)=G(X, Y)$ if and only if $A^{2}+B^{2}=1$.

Now, we consider the 2 -form $\Omega(X, Y)=G(X, J Y)$.

Lemma 4.2. $\Omega$ is closed if and only if

$$
A y^{l}\left(-\stackrel{g}{R}{ }_{i j l}^{m} J_{m k}+\stackrel{g}{R}{ }_{i k l}^{m} J_{m j}-\stackrel{g}{R}{ }_{j k l}^{m} J_{m i}\right)=0,
$$

and

$$
B_{\tau}\left(y_{j} g_{i k}-y_{k} g_{i j}\right)=A_{\tau}\left(y_{j} J_{k i}-y_{k} J_{j i}\right) .
$$

Proof. It is easy to see that

$$
\begin{gathered}
\mathrm{d} \Omega\left(\partial_{\bar{i}}, \partial_{\bar{j}}, \partial_{\bar{k}}\right)=0=\mathrm{d} \Omega\left(\delta_{i}, \delta_{j}, \partial_{\bar{k}}\right), \\
\mathrm{d} \Omega\left(\delta_{i}, \delta_{j}, \delta_{k}\right)=A y^{l}\left(-\stackrel{g}{R_{i j l}^{m}} J_{m k}+\stackrel{g}{R_{i k l}^{m}} J_{m j}-\stackrel{g}{R_{j k l}^{m}} J_{m i}\right),
\end{gathered}
$$

and

$$
\mathrm{d} \Omega\left(\delta_{i}, \partial_{\bar{j}}, \partial_{\bar{k}}\right)=B_{\tau}\left(y_{j} g_{i k}-y_{k} g_{i j}\right)+A_{\tau}\left(y_{k} J_{j i}-y_{j} J_{k i}\right) .
$$

The above equations complete the proof.

Lemma 4.3. The almost complex structure $\bar{J}$ is integrable if and only if

$$
\begin{array}{r}
B B_{\tau} y_{i} \delta_{j}^{n}+B A_{\tau} y_{i} J_{j}^{n}+A J_{i}^{m} B_{\tau} y_{m} \delta_{j}^{n}+A J_{i}^{m} J_{j}^{n} A_{\tau} y_{m}-B B_{\tau} y_{j} \delta_{i}^{n} \\
-B A_{\tau} y_{j} J_{i}^{n}-A J_{j}^{m} B_{\tau} y_{m} \delta_{i}^{n}-A A_{\tau} J_{j}^{m} J_{i}^{n} y_{m}+y^{m} \stackrel{g}{R_{i j m}^{n}}=0 .
\end{array}
$$


Proof. Using the property of $\bar{J}$, we can derive that $N_{\bar{J}}\left(\partial_{\bar{i}}, \partial_{\bar{j}}\right)=N_{\bar{J}}\left(\delta_{i}, \partial_{\bar{j}}\right)=0$ if and only if $N_{\bar{J}}\left(\delta_{i}, \delta_{j}\right)=0$ (see [14] for details). Therefore, it is sufficient to obtain $N_{\bar{J}}\left(\delta_{i}, \delta_{j}\right)$. To this aim, we use the straightforward equality

$$
\begin{aligned}
& N_{\bar{J}}\left(\delta_{i}, \delta_{j}\right)=\left\{B B_{\tau} y_{i} \delta_{j}^{n}+B A_{\tau} y_{i} J_{j}^{n}+A J_{i}^{m} B_{\tau} y_{m} \delta_{j}^{n}+A J_{i}^{m} J_{j}^{n} A_{\tau} y_{m}-B B_{\tau} y_{j} \delta_{i}^{n}\right. \\
& \left.\quad-B A_{\tau} y_{j} J_{i}^{n}-A J_{j}^{m} B_{\tau} y_{m} \delta_{i}^{n}-A A_{\tau} J_{j}^{m} J_{i}^{n} y_{m}+y^{m} \underset{R_{i j m}^{n}}{n}\right\} \partial_{\bar{n}} .
\end{aligned}
$$

Theorem 4.4. Let $(M, g, J)$ be a Kähler manifold. Then $(\mathrm{T} M, G, \bar{J})$ is a Kähler manifold if and only if (4.2)-(4.5) hold.

Corollary 4.5. Let $(M, g, J)$ be a flat Kähler manifold and let $A$ and $B$ be constant functions with respect to $\tau$, satisfying in $A^{2}+B^{2}=1$. Then $(\mathrm{T} M, G, \bar{J})$ is a Kähler manifold.

Definition 4.6. [11] A triple $(\nabla, g, J)$ is called a holomorphic statistical structure on $M$ if $(\nabla, g)$ is a statistical structure, $(g, J)$ is a Kähler structure on $M$, and the formula

$$
K_{X} J Y+J K_{X} Y=0,
$$

holds for any $X, Y \in \Gamma(\mathrm{T} M)$.

Remark 4.7. We note that (4.6) induces $J_{j}^{m} K_{i m}^{s}+K_{i j}^{m} J_{m}^{s}=0$, where

$$
K_{i j}^{m}=\Gamma_{i j}^{m}-\stackrel{g}{\Gamma}{ }_{i j}^{m} .
$$

Lemma 4.8. The difference tensor field $\bar{K}=\bar{\nabla}-\stackrel{g}{\nabla}$, has its components

explicitly given by:

$$
\begin{aligned}
& \bar{K}\left(\delta_{i}, \delta_{j}\right)=\bar{\nabla}_{\delta_{i}} \delta_{j}-\stackrel{g}{\nabla}_{\delta_{i}} \delta_{j}=\bar{K}_{i j}^{k} \delta_{k}+\bar{K}_{i j}^{\bar{k}} \partial_{\bar{k}}, \\
& \bar{K}\left(\delta_{i}, \partial_{\bar{j}}\right)=\bar{\nabla}_{\delta_{i}} \partial_{\bar{j}}-\stackrel{\underline{\nabla}}{\nabla}_{\delta_{i}} \partial_{\bar{j}}=\bar{K}_{i \bar{j}}^{k} \delta_{k}+\bar{K}_{i \bar{j}}^{\bar{k}} \partial_{\bar{k}}, \\
& \bar{K}\left(\partial_{\bar{i}}, \partial_{\bar{j}}\right)=\bar{\nabla}_{\partial_{\bar{i}}} \partial_{\bar{j}}-{\stackrel{\underline{\nabla}}{\partial_{\bar{i}}}}_{\partial_{\bar{j}}}=\bar{K} \frac{k}{i j} \delta_{k}+\bar{K}_{\bar{i}}^{\underline{k}} \partial_{\bar{k}} .
\end{aligned}
$$

$$
\begin{aligned}
\bar{K}_{i j}^{k} & =\bar{\Gamma}_{i j}^{k}-\Gamma_{i j}^{k}, \\
\bar{K}_{i j}^{\bar{k}} & =\bar{\Gamma}_{i j}^{\bar{k}}+\frac{1}{2} y^{l} \stackrel{g}{R_{i j l}^{k},} \\
\bar{K}_{i j}^{k} & =\bar{\Gamma}_{i \bar{j}}^{k}-\frac{1}{2} y^{l} \stackrel{g}{R_{l j i}^{k},} \\
\bar{K}_{i j}^{\bar{k}} & =\bar{\Gamma}_{\bar{j}}^{\bar{k}}, \\
\bar{K}_{\overline{i j}}^{k} & =\bar{\Gamma}_{\overline{i j}}^{k}, \\
\bar{K}_{\overline{i j}}^{\bar{k}} & =\bar{\Gamma}_{\overline{i j}}^{\bar{k}} .
\end{aligned}
$$

Theorem 4.9. The components of the difference tensor field $\bar{K}$ satisfy the following relations:

$$
\begin{aligned}
& \bar{K}_{i j}^{k}=K_{i j}^{k}+\bar{\Gamma}_{i j}^{k}-{ }^{g} \Gamma_{i j}^{k}, \\
& \left(\bar{K}_{i k}^{r}-K_{i k}^{r}\right) g_{r j}=\left(\bar{K}_{j k}^{r}-K_{j k}^{r}\right) g_{r i}, \\
& \bar{K}_{i \bar{k}}^{\bar{r}} g_{j r}=\bar{K}_{j \bar{k}}^{r} g_{r i}, \\
& \bar{K}_{\bar{i} \bar{k}}^{\bar{r}} g_{r j}=\bar{K}_{\bar{j} \bar{k}}^{\bar{r}} g_{r i},
\end{aligned}
$$




$$
\begin{aligned}
\bar{K}_{i \bar{j}}^{\bar{r}} g_{r k} & =\bar{K}_{i \bar{k}}^{\bar{r}} g_{r j}, \\
\bar{K}_{i \bar{k}}^{r} g_{j r} & =\bar{K}_{j \bar{k}}^{r} g_{r i}, \\
\bar{K}_{i j}^{\bar{r}} g_{r k} & =\bar{K}_{j \bar{k}}^{r} g_{r i} .
\end{aligned}
$$

Proof. From (4.8) and (4.7), we obtain (4.14). Also (4.14) and (3.5) give (4.15). Using (4.11), (4.12) and (3.8), we get (4.16). (4.13) and (3.10) imply (4.17). Also, (4.11) and (3.9) imply (4.18). (4.10) and (3.7) give (4.19). Moreover, (4.9), (4.10) and (3.6) imply (4.20).

We further study the holomorphic conditions for connections $\bar{\nabla}$, where $(G, \bar{\nabla})$ is a statistical structure on the tangent bundle endowed with Sasaki metric $G$. To this aim, we need to check the equation (4.6); direct calculations lead to:

$$
\begin{aligned}
\bar{K}_{\delta_{i}} \bar{J} \delta_{j}+\bar{J} \bar{K}_{\delta_{i}} \delta_{j}= & {\left[B\left(\bar{\Gamma}_{i \bar{j}}^{s}-\frac{1}{2} y^{l} \stackrel{g}{R_{l j i}^{s}}\right)+A J_{j}^{m}\left(\bar{\Gamma}_{i \bar{m}}^{s}-\frac{1}{2} y^{l} \stackrel{g}{R_{l m i}^{s}}\right)-B\left(\bar{\Gamma}_{i j}^{\bar{s}}+\frac{1}{2} y^{l} \stackrel{g}{R_{i j l}^{s}}\right)\right.} \\
& \left.+A J_{n}^{s}\left(\bar{\Gamma}_{i j}^{\bar{n}}+\frac{1}{2} y^{l} \stackrel{g}{R_{i j l}^{n}}\right)\right] \delta_{s}+\left[B \bar{\Gamma}_{\bar{j} i}^{\bar{s}}+A J_{j}^{m} \bar{\Gamma}_{\bar{m} i}^{\bar{s}}+B\left(\bar{\Gamma}_{i j}^{s}-\stackrel{\Gamma}{\Gamma}_{i j}^{s}\right)\right. \\
& \left.+A J_{n}^{s}\left(\bar{\Gamma}_{i j}^{n}-\stackrel{g}{\Gamma}_{i j}^{n}\right)\right] \partial_{\bar{s}},
\end{aligned}
$$

Theorem 4.10. Let $(M, g, J)$ be a Kähler manifold, let $(g, \nabla)$ be a statistical structure on $M$, and $(G, \bar{\nabla})$ be a statistical structure on $\mathrm{T} M$. Then $(\mathrm{T} M, G, \bar{J}, \bar{\nabla})$ is holomorphic 
if and only if (4.2)-(4.5) hold and, moreover,

$$
\begin{aligned}
& B\left(\bar{\Gamma}_{i \bar{j}}^{s}-\frac{1}{2} y^{l} \stackrel{g}{R_{l j i}^{s}}\right)+A J_{j}^{m}\left(\bar{\Gamma}_{i \bar{m}}^{s}-\frac{1}{2} y^{l} \stackrel{g}{R_{l m i}^{s}}\right) \\
& -B\left(\bar{\Gamma}_{i j}^{\bar{s}}+\frac{1}{2} y^{l} \stackrel{g}{R_{i j l}^{s}}\right)+A J_{n}^{s}\left(\bar{\Gamma}_{i j}^{\bar{n}}+\frac{1}{2} y^{l} \stackrel{g}{R_{i j l}^{n}}\right)=0, \\
& B \bar{\Gamma} \frac{\bar{s}}{j i}+A J_{j}^{m} \bar{\Gamma}_{\bar{m} i}^{\bar{s}}+B\left(\bar{\Gamma}_{i j}^{s}-\stackrel{g}{\Gamma}{ }_{i j}^{s}\right)+A J_{n}^{s}\left(\bar{\Gamma}_{i j}^{n}-\stackrel{g}{\Gamma}{ }_{i j}^{n}\right)=0, \\
& -B\left(\bar{\Gamma}_{\bar{i} j}^{s}-\frac{1}{2} y^{l} \stackrel{g}{R_{l i j}^{s}}\right)+A J_{j}^{m}\left(\bar{\Gamma}_{\bar{i} m}^{s}-\frac{1}{2} y^{l} \stackrel{g}{R_{l i m}^{s}}\right)+\left(-B \bar{\Gamma}_{\bar{i} \bar{j}}^{\bar{s}}+A \bar{\Gamma}_{\bar{i} \bar{j}}^{\bar{m}} J_{m}^{s}\right)=0, \\
& -B \bar{\Gamma} \overline{\bar{i} j}+A J_{j}^{m} \bar{\Gamma}_{\bar{i} m}^{\bar{s}}+B \bar{\Gamma} \bar{i}_{\bar{i} j}^{s}+A \bar{\Gamma}_{\bar{i} \bar{j}}^{m} J_{m}^{s}=0, \\
& -B\left(\bar{\Gamma}_{i j}^{s}-\stackrel{g}{\Gamma}{ }_{i j}^{s}\right)+A J_{j}^{m}\left(\bar{\Gamma}_{i m}^{s}-\stackrel{g}{\Gamma_{i m}^{s}}\right)-B \bar{\Gamma}_{\bar{j} i}^{\bar{s}}+A J_{m}^{s} \bar{\Gamma}_{\bar{j} i}^{\bar{m}}=0, \\
& -B\left(\bar{\Gamma}_{i j}^{\bar{s}}+\frac{1}{2} y^{l} \stackrel{g}{R_{i j l}^{s}}\right)+A J_{j}^{m}\left(\bar{\Gamma}_{i m}^{\bar{s}}+\frac{1}{2} y^{l} \stackrel{g}{R_{i m l}^{s}}\right) \\
& +B\left(\bar{\Gamma}_{i \bar{j}}^{s}-\frac{1}{2} y^{l} \stackrel{g}{R_{l j i}^{s}}\right)+A J_{m}^{s}\left(\bar{\Gamma}_{i \bar{j}}^{m}-\frac{1}{2} y^{l} \stackrel{g}{R_{l j i}^{m}}\right)=0 . \\
& B \bar{\Gamma} \overline{\bar{i}}_{\bar{j}}^{s}+A J_{j}^{m} \bar{\Gamma}_{\bar{i} \bar{m}}^{s}-B \bar{\Gamma}_{\bar{i} j}^{\bar{s}}+A J_{m}^{s} \bar{\Gamma}_{\bar{i} j}^{\bar{m}}=0 \text {, } \\
& B \bar{\Gamma}_{\bar{i} \bar{j}}^{\bar{s}}+A J_{j}^{m} \bar{\Gamma}_{\bar{i} \bar{m}}^{\bar{s}}+B\left(\bar{\Gamma}_{j \bar{i}}^{s}-\frac{1}{2} y^{l} \stackrel{g}{R_{l i j}^{s}}\right)+A J_{m}^{s}\left(\bar{\Gamma}_{j \bar{i}}^{m}-\frac{1}{2} y^{l} \stackrel{g}{R_{l i j}^{m}}\right)=0 .
\end{aligned}
$$

Theorem 4.11. Let $(M, g, J)$ be a Kähler manifold, let $(g, \nabla)$ be a statistical structure on $M$, and let $(G, \bar{\nabla})$ be the statistical structure on TM given by Example 3.4. Then $(\mathrm{T} M, G, \bar{J}, \bar{\nabla})$ is holomorphic if and only if (4.2)-(4.5) hold and, moreover, $\bar{\nabla}$ reduces to the Levi-Civita connection of $G$.

Proof. Using Theorems 4.4 and 4.10, it is easy to check that $(\mathrm{T} M, G, \bar{J}, \bar{\nabla})$ is holomorphic if and only if (4.2)-(4.5) hold, and

$$
\begin{aligned}
A\left(J_{j}^{m} g^{s r} C_{m r i}+J_{m}^{s} g^{m r} C_{j r i}\right) & =0, \\
B g^{s r} C_{j r i}+A J_{j}^{m} g^{s r} C_{m r i} & =0, \\
B g^{s r} C_{j r i}-A J_{j}^{m} g^{s r} C_{m r i} & =0 .
\end{aligned}
$$

(4.21) and (4.22) imply $B g^{s r} C_{j r i}=0$. If $B \neq 0$, then we deduce $C_{j r i}=0$, i.e., $\bar{\nabla}$ reduces to the Levi-Civita connection. But, if $B=0$, then from (4.21) we conclude $J_{j}^{m} g^{s r} C_{m r i}=0$. We note that in this case we have $A \neq 0$. By multiplying this equations with $J_{h}^{j} g_{s n}$, we infer $C_{h n i}=0$. Thus, in this case, $\bar{\nabla}$ reduces to the Levi-Civita connection as well.

Corollary 4.12. Let $(M, g, J)$ be a flat Kähler manifold, let $A$ and $B$ be constant functions with respect to $\tau$ satisfying the condition $A^{2}+B^{2}=1$, let $(g, \nabla)$ be a statistical structure on $M$, and let $(G, \bar{\nabla})$ be the statistical structure on $\mathrm{T} M$ given by Example 3.4. Then $(\mathrm{T} M, G, \bar{J}, \bar{\nabla})$ is holomorphic if and only if $\bar{\nabla}$ reduces to the Levi-Civita connection of $G$.

Proposition 4.13. The Riemannian manifold introduced in Example 3.8 carries only two Kähler structures

$$
\left\{\begin{array}{l}
J_{1}^{1}=J_{2}^{2}=0, \quad J_{1}^{2}= \pm \frac{1}{\sqrt{2}}, \quad J_{2}^{1}=\mp \sqrt{2}, \\
\omega_{11}=\omega_{22}=0, \quad \omega_{21}=\sqrt{2} \frac{1}{x_{2}^{2}}=-\omega_{12} .
\end{array}\right.
$$

Proof. The first relation in (4.1) leads to

$$
\left(J_{1}^{1}\right)^{2}+J_{1}^{2} J_{2}^{1}=\left(J_{2}^{2}\right)^{2}+J_{1}^{2} J_{2}^{1}=-1, \quad J_{1}^{1} J_{1}^{2}+J_{2}^{2} J_{1}^{2}=J_{1}^{1} J_{2}^{1}+J_{2}^{2} J_{2}^{1}=0 .
$$

The second relation in (4.1) leads to

$$
\left\{\begin{array}{l}
J_{1}^{1} J_{2}^{1}+2 J_{2}^{2} J_{1}^{2}=J_{1}^{1} J_{2}^{1}+2 J_{2}^{2} J_{1}^{2}=0 \\
\left(J_{1}^{1}\right)^{2}+2\left(J_{1}^{2}\right)^{2}=1, \quad\left(J_{2}^{1}\right)^{2}+2\left(J_{2}^{2}\right)^{2}=2
\end{array}\right.
$$


From (4.24) and (4.25), it results that the only possible situations for $J$ to satisfy the first two equations in (4.1) are the ones described in (4.23). Further, the third relation in (4.1) allows to find the coefficients of $\omega$, as described in (4.23). Also, it is easy to check that the other relations in (4.1) can be achieved by (4.23).

Proposition 4.14. Let $\left(\mathbb{R}^{2}, g, J\right)$ be the Kähler manifolds introduced in Proposition 4.13. Then, $\left(\mathrm{TR}^{2}, G, \bar{J}\right)$ is an almost Kähler manifold if and only if $A$ and $B$ are constant functions with respect to $\tau$.

Proof. It is sufficient to verify the relations (4.3) and (4.4). Straight computations lead to (4.3). If $A$ and $B$ are constants, then (4.4) shall be obtained. Otherwise, putting $i=$ $j=1, k=2$ and $i=j=2, k=2$ in (4.4) leads to the meaningless relation $y_{2}^{2}=-2 y_{1}^{2}$.

Theorem 4.15. The structure $\left(\mathrm{TR}^{2}, G, \bar{J}\right)$ mentioned in Proposition 4.14 cannot be a Kähler one.

Proof. Indeed, we must show that $\bar{J}$ cannot be integrable. Since $A$ and $B$ are constant functions with respect to $\tau$, (4.5) reduces to $y^{m} \stackrel{g}{R_{i j m}^{n}}=0$. The above relation contradicts the non-flatness of the upper half-space of the Poincare plane.

Proposition 4.16. The Riemannian manifold introduced in Example 3.9 carries only two Kähler structures,

$$
\left\{\begin{array}{l}
J_{1}^{1}=J_{2}^{2}=0, \quad J_{1}^{2}= \pm 1, \quad J_{2}^{1}=\mp 1 \\
\omega_{11}=\omega_{22}=0, \quad \omega_{21}=1=-\omega_{12}
\end{array}\right.
$$

Proof. Similar to the proof of Proposition 4.13.

Proposition 4.17. Let $\left(\mathbb{E}^{2}, g, J\right)$ be the Kähler manifolds introduced in Proposition 4.16. Then, $\left(\mathrm{TE}^{2}, G, \bar{J}\right)$ is a Kähler manifold if and only if $A$ and $B$ are constant functions with respect to $\tau$.

Proof. We check first (4.4). By putting $i=j=1, k=2$ and $i=j=k=2$ in (4.4), it results $B_{\tau} y_{2}=A_{\tau} y_{1}$ and $B_{\tau} y_{1}=-A_{\tau} y_{2}$ respectively. From the two latter ones, one can deduce that $A_{\tau} y_{1}^{2}=-A_{\tau} y_{1}^{2}$, and hence $A$ is constant; thus, $B$ is constant as well. Because the curvature is zero, it follows that (4.3) and (4.5) hold.

Example 4.18. We consider the Kähler structure $\left(\mathrm{TE}^{2}, G, \bar{J}\right)$ with $A=0, B=1$ and fix the connection $\bar{\nabla}$ with the coefficients

$$
\left\{\begin{array}{l}
\bar{\Gamma}_{i j}^{s}=-\bar{\Gamma}_{\bar{j} i}^{\bar{s}}=-f\left(x_{1}, x_{2}, y_{1}, y_{2}\right), \\
\bar{\Gamma}_{\bar{i}}^{s}=\bar{\Gamma}_{\bar{i} j}^{\bar{s}}=f\left(x_{1}, x_{2}, y_{1}, y_{2}\right), \\
\bar{\Gamma}_{\bar{i} \bar{s}}^{\bar{s}}=\bar{\Gamma}_{\bar{i} j}^{s}=\bar{\Gamma}_{i \bar{j}}^{s}=0,
\end{array}\right.
$$

for all $i, j, s \in\{1,2\}$. It is easy to see that $(G, \bar{\nabla})$ is a statistical structure on $\mathrm{TE}^{2}$, and it satisfies the relations from the Theorem 4.10. Therefore, $\left(\mathrm{TE}^{2}, G, \bar{J}, \bar{\nabla}\right)$ is a holomorphic manifold.

\section{References}

[1] S. Amari, Information geometry of the EM and em algorithms for neural networks, Neural Networks, 8 (9), 1379-1408, 1995.

[2] S. Amari and H. Nagaoka, Methods of information geometry, American Mathematical Society, 2000.

[3] V. Balan, H.V. Grushevskaya,N.G. Krylova, G.G. Krylov, and I.V. Lipnevich, Twodimensional first-order phase transition as signature change event in contact statistical manifolds with Finsler metric, Appl. Sci. 21, 11-26, 2019. 
[4] M. Belkin, P. Niyogi and V.Sindhwani, Manifold regularization: a geometric framework for learning from labeled and unlabeled examples, J. Mach. Learn. Res. 7, 23992434, 2006.

[5] M. Burgin, Theory of information: fundamentality, diversity and unification, World Scientific Series in Information Studies, 2009.

[6] A. Caticha, Geometry from information geometry, https://arxiv.org/abs/1512. 09076v1.

[7] A. Caticha, The information geometry of space and time, https://arxiv.org/abs/ gr-qc/0508108.

[8] N.N. Cencov, Statistical decision rules and optimal inference, Amer. Mathematical Society: Translations of Mathematical Monographs, 1982.

[9] R.A. Fisher, On the mathematical foundations of theoretical statistics, Philos. Trans. R. Soc. Lond. Ser. A Math. Phys. Eng. Sci. 222, 309-368, 1922.

[10] H. Furuhata, Hypersurfaces in statistical manifolds, Diff. Geom. Appl. 27, 420-429, 2009.

[11] H. Furuhata, I. Hasegawa, Y. Okuyama, K. Sato and M.H. Shahid, Sasakian statistical manifolds, J. Geom. Phys. 117, 179-186, 2017.

[12] S. Ianus, Statistical manifolds and tangent bundles, Sci. Bull. Univ. Politechnica of Bucharest Ser. D, 56, 29-34, 1994.

[13] S. Lauritzen, Statistical manifolds, in: Differential Geometry in Statistical Inference, IMS Lecture Notes 10, 163-216, 1987.

[14] H. Matsuzoe and J.I. Inoguchi, Statistical structures on tangent bundles, APPS. Appl. Sci. 5 (1), 55-57, 2003.

[15] J.M. Oller and C.N. Caudras, Rao's distance for negative multinomial distributions, Sankhya A, 47 (1), 75-83, 1985.

[16] C.R. Rao, Information and accuracy attainable in estimation of statistical parameters, Bull. Cal. Math. Soc. 37, 81-91, 1945.

[17] K. Sun and S. Marchand-Maillet, An information geometry of statistical manifold learning, Proceedings of the 31st International Conference on Machine Learning (ICML-14), 1-9, 2014.

[18] K. Yano and S. Ishihara, Tangent and cotangent bundles, Marcel Dekker Inc., New York, 1973. 